\title{
The updated version of the Chinese Evaluated Nuclear Data Library (CENDL-3.1) and China nuclear data evaluation activities
}

\author{
Ge Zhigang ${ }^{1, a}$, Yu Hongwei ${ }^{1}$, Zhuang Youxiang ${ }^{1}$, Liu Tingjin ${ }^{1}$, Zhang Jingshang ${ }^{1}$, Liu Ping ${ }^{1}$, Huang Xiaolong ${ }^{1}$, \\ Zhao Zhixiang $^{2}$, and Xia Haihong ${ }^{3}$ \\ 1 China Nuclear Data Center, China Institute of Atomic Energy (CIAE), Beijing 102413, P.R. China \\ 2 China Committee of Nuclear Data, China Institute of Atomic Energy (CIAE), Beijing 102413, P.R. China \\ 3 The Department of Nuclear Physics, China Institute of Atomic Energy (CIAE), Beijing 102413, P.R. China
}

\begin{abstract}
Chinese Evaluated Nuclear Data Library third version (CENDL-3.1) contains the nuclear data evaluated works which were performed during the period of 1995-2005 at China Nuclear Data Center (CNDC) in cooperation with the China Nuclear Data Coordination Network (CNDCN), including the neutron data for about 200 nuclides in ENDF/B-6 format in energy region of $10^{-5} \mathrm{eV}-20 \mathrm{MeV}$. For the most important nuclei the comparisons with other nuclear data libraries (ENDF, JENDL, BROND, JEFF...) have been performed, and the benchmark testing for the most important nuclei also have been done. CNDC and CNDCN also have got a lot of progress in the fields of nuclear data theory study, model and code developments, and nuclear database establishment, etc. The status of CENDL-3 and some progress of the nuclear data evaluation studies in the past several years will be introduced in this presentation.
\end{abstract}

\section{CENDL-3.1 Library}

\subsection{The view of CENDL-3.1}

The evaluated activities of CENDL-3.1 were started in 1995 and finished in 2005. The library includes comprehensive data evaluations for all neutron reactions in the energy range from $10^{-5} \mathrm{eV}$ to $20 \mathrm{MeV}$ for 200 nuclides, among which 133 nuclides are newly evaluated, and 67 nuclides are taken from CENDL-2.1.

The experimental data involved into the evaluations were taken from the EXFOR library, the literary indexed by CINDA and the experimental data measured in China domestically institutes and universities. The experimental data were evaluated carefully and corrected by using the new standard cross sections and decay data et al.

UNF [1] series codes LUNF, UNF, FUNF [2] were used in the model calculations for the light elements, structural materials, fission products and medium elements, heavy elements and actinides, respectively. Most of the input model parameters are taken from the RIPL [3] library and were adjusted based on the experimental information. The APMN [4] and APOM94 [5] programs are used for optimal optical potential parameters automatically searching. ECIS95 [6] is also involved for some model calculations.

All evaluations were checked by using the ENDF facilities CHECKR, PHYCHE and FIZCON.

CENDL-3.1 was distributed inside of CNDCN and some users in China as an internal test version in 2001. After that some feedback information from benchmark testing and users has been received. These were considered in the re-evaluations for improving the data of the important nuclides, especially for the uranium and plutonium isotopes when the improvement is completed, a new version of CENDL-3.0, CENDL-3.1 will be released.

\footnotetext{
${ }^{a}$ Presenting author, e-mail: gezgl@ciae.ac.cn
}

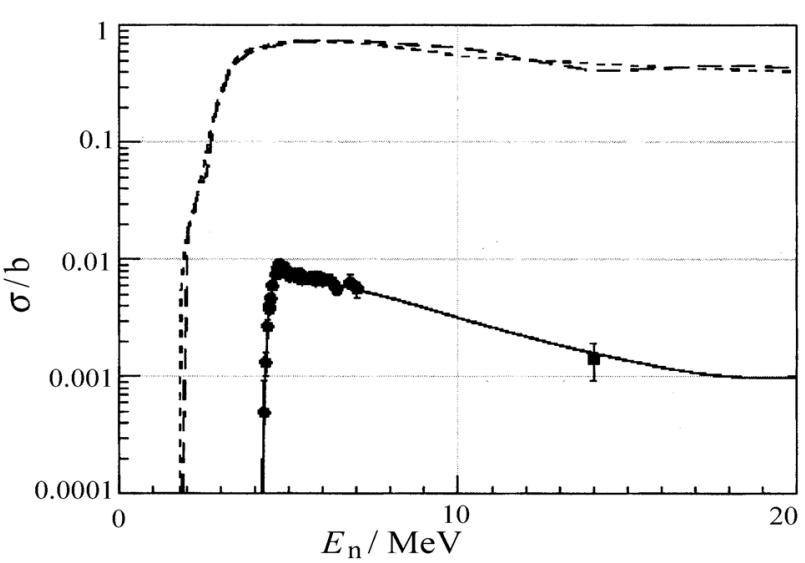

Fig. 1. ${ }^{6} \mathrm{Li}\left(\mathrm{n}, \mathrm{n}^{\prime} \gamma\right)^{6} \mathrm{Li}$ total inelastic scattering reaction.

\subsection{CENDL-3.1 library description}

\section{Light elements}

Based on the updated experimental information, a series of codes (LUNF) were developed for model calculations of ${ }^{6,7} \mathrm{Li}$, ${ }^{9} \mathrm{Be},{ }^{10,11} \mathrm{~B},{ }^{12} \mathrm{C},{ }^{14} \mathrm{~N}$ and ${ }^{16} \mathrm{O}$, mainly for double differential cross sections (MF-6) [7]. The full sets of neutron data, i.e., cross sections of all reactions, energy and angular distributions of secondary neutrons and differential cross sections, etc. were provided.

\section{Structural materials}

According to the MUP [8] code used in the CENDL-2 evaluations and the new results of nuclear reaction theoretical studies, a new code UNF used for structural material and medium nuclides calculation was developed. With the UNF code calculations and evaluation of new experimental data, the full neutron data set ( $\gamma$-production data including) are provided in ENDF/B-6 format. 
Table 1. The nuclides of CENDL-3.1.

\begin{tabular}{|c|c|}
\hline Nucl. & Content \\
\hline Light Elements & ,2,3 $\mathrm{H},{ }^{3,4} \mathrm{He},{ }^{6,7} \mathrm{Li},{ }^{9} \mathrm{Be},{ }^{10,11} \mathrm{~B},{ }^{12} \mathrm{C},{ }^{14} \mathrm{~N},{ }^{16} \mathrm{O},{ }^{19} \mathrm{~F},{ }^{23} \mathrm{Na},{ }^{\text {nat }} \mathrm{Mg},{ }^{27} \mathrm{Al},{ }^{\text {nat }} \mathrm{Si},{ }^{31} \mathrm{P},{ }^{\text {nat }} \mathrm{S},{ }^{\text {nat }} \mathrm{Cl},{ }^{\text {nat }} \mathrm{K},{ }^{\text {nat }} \mathrm{Ca}$ \\
\hline Structural Materials & ${ }^{\text {nat }} \mathrm{Ti}^{\text {,nt }}{ }^{\text {na }},{ }^{50,52,53,54, \text { nat }} \mathrm{Cr},{ }^{55} \mathrm{Mn},{ }^{54,56,57,58, \text { nat }} \mathrm{Fe},{ }^{59} \mathrm{Co},{ }^{58,60,61,62,64, \text { nat }} \mathrm{Ni},{ }^{63,65, \text { nat }} \mathrm{Cu},{ }^{\text {nat }} \mathrm{Zn}$ \\
\hline $\begin{array}{l}\text { Fission Products \& } \\
\text { Medium Elements }\end{array}$ & 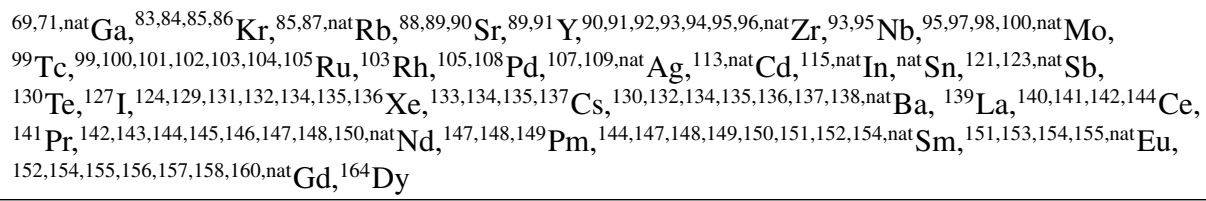 \\
\hline Heavy Elements & ${ }^{\text {nat }} \mathrm{Lu},{ }^{\text {nat }} \mathrm{Hf},{ }^{181} \mathrm{Ta},{ }^{\text {nat }} \mathrm{W},{ }^{197} \mathrm{Au},{ }^{\text {nat }} \mathrm{Hg},{ }^{\text {nat }} \mathrm{Ti},{ }^{204,206,207, \text { nat }} \mathrm{Pb}$ \\
\hline Actinides & ${ }^{233,234,235,236,238,239} \mathrm{U},{ }^{237} \mathrm{~Np},{ }^{238,239,240,241,242} \mathrm{Pu},{ }^{241,242} \mathrm{Am},{ }^{249} \mathrm{Bk},{ }^{249} \mathrm{Cf}$ \\
\hline
\end{tabular}

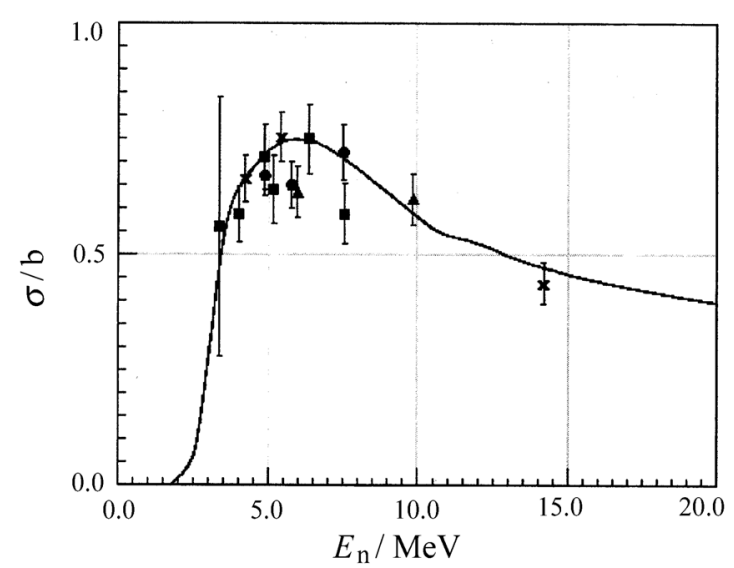

Fig. 2. ${ }^{6} \mathrm{Li}(\mathrm{n}, \mathrm{nd}){ }^{4} \mathrm{He}$ reaction cross section.

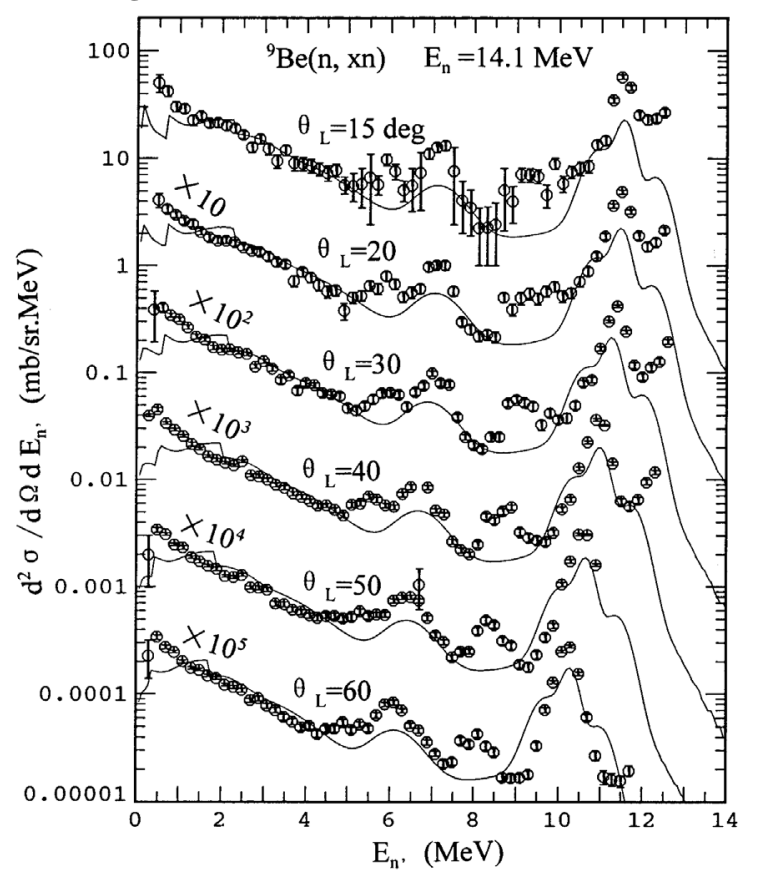

Fig. 3. The double differential cross sections (MF-6) of ${ }^{9} \mathrm{Be}$, comparison with experimental data.

Comparing to CENDL-2, the main development of CENDL-3.1 for the structured materials is that in addition to the data of natural elements, the data of their isotopes were also included, and we made the data consistent between the natural elements and their isotopes.

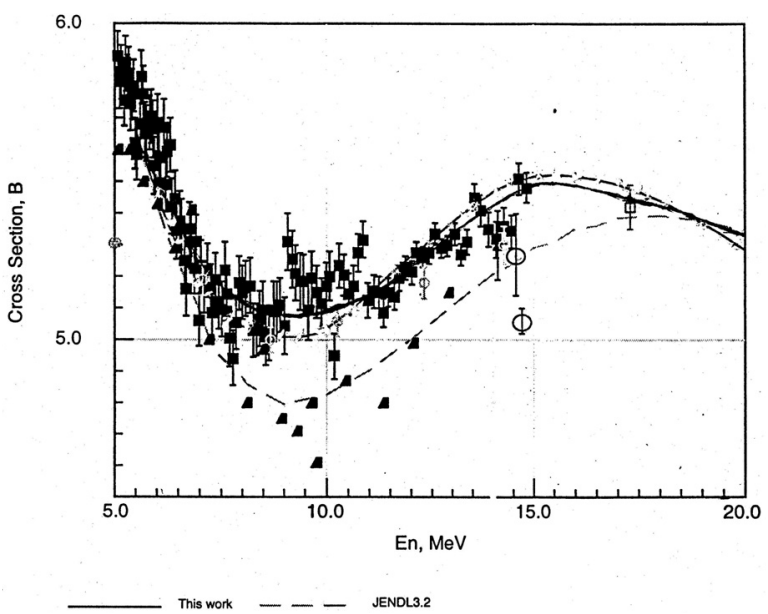

Fig. 4. ${ }^{\text {nat }} \mathrm{W}(\mathrm{n}$,tot $)$ comparison with experimental data and JENDL3.2.

\section{Fission products}

Files MF-1 to MF-5 were provided in CENDL-3.1 for most fission products nuclides, MF-1 to MF-6 are variable for others. A code SUNF, the simple version of UNF, was developed for the model calculations of fission products nuclides. The data of 101 fission products nuclides were sent to be used in the international comparison of FP and coordinated by the NEA/WPEC Subgroup 21, and 38 of them were selected as the data file of release 7 of the ENDF/B library.

\section{Actinides}

On the basis of the model calculations with the code FUNF and adjusting the model parameters carefully, the new experimental data, 15 actinides were evaluated or re-evaluated. MF-1 6, $12 \sim 15$ were included for important actinides (i.e., $\mathrm{U}, \mathrm{Pu}$ isotopes), and MF-1 $\sim 5$ for others. The file number and nuclides were extended comparing with the previous version of CENDL, and the results of the benchmark testing have been considered during the evaluation process.

\subsection{The benchmark testing of CENDL-3.1}

In order to test the reliability of the data from CENDL-3.1, the benchmark testing for some light, medium-heavy nuclides 


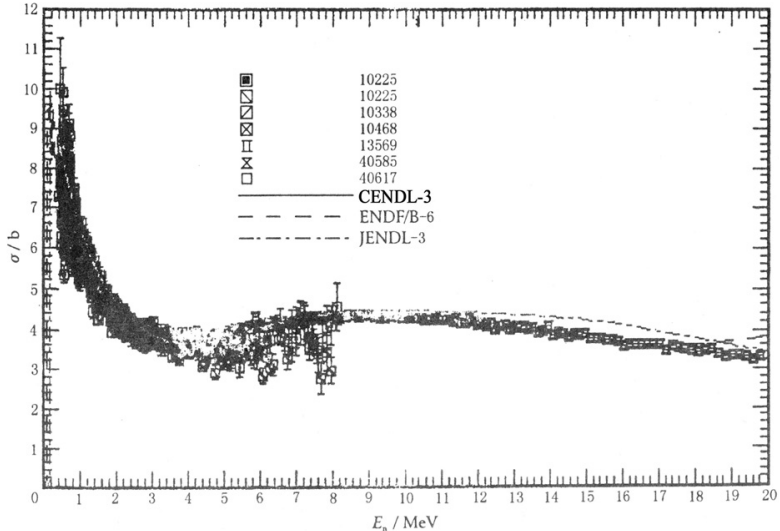

Fig. 5. The evaluated total cross section of $\mathrm{n}+{ }^{90} \mathrm{Zr}$ compared with experimental data and JENDL3.2, ENDF/B-VI.

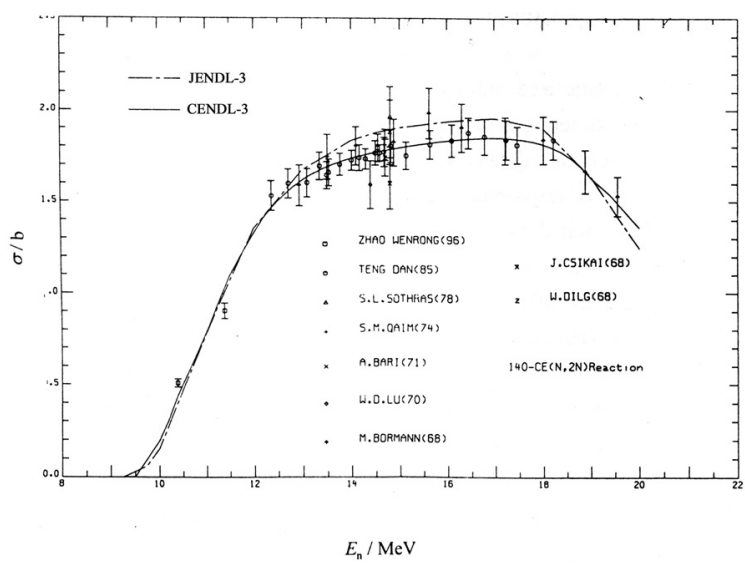

Fig. 6. The evaluated $(n, 2 n)$ reaction cross section of $n+{ }^{140} \mathrm{Ce}$.

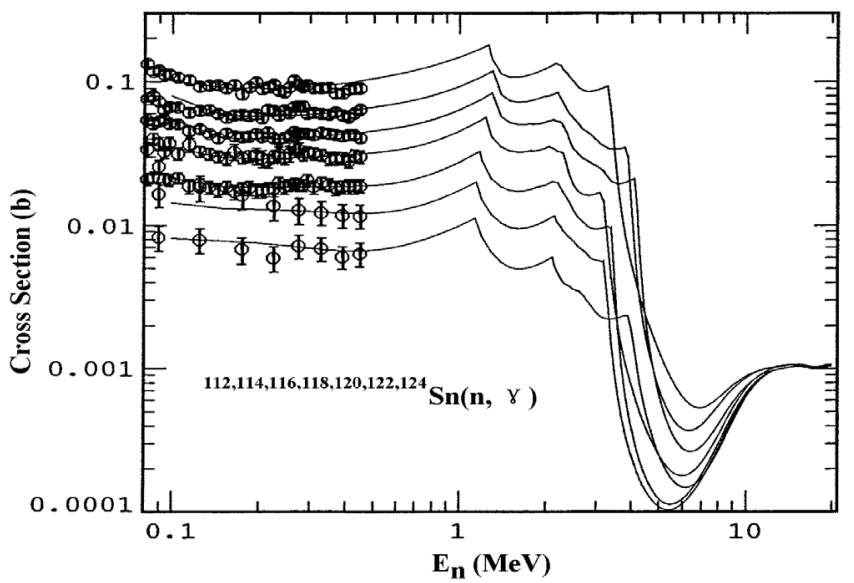

Fig. 7. $\operatorname{Sn}(\mathrm{n}, \gamma)$ reaction evaluation compared with experimental data.

and actinides of CENDL-3.1 has been performed. The calculations and analyses of benchmarks were done with the MCNP Monte Carlo code and transformation codes. The data processing was carried out by using the internationally used code system NJOY94. These benchmarks were based on IAEA specifications [9] for the FNS (Fusion Neutronics Source) experiments [10] and OKTAVIAN experiments [11] in Japan. Some results were compared with ENDF/B-VI,

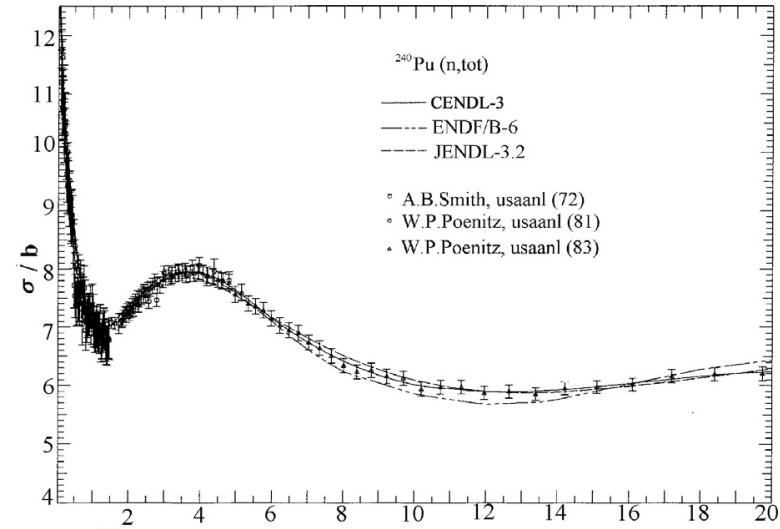

Fig. 8. ${ }^{240} \mathrm{Pu}(\mathrm{n}$,tot $)$ evaluations compared with experimental data and other libraries.

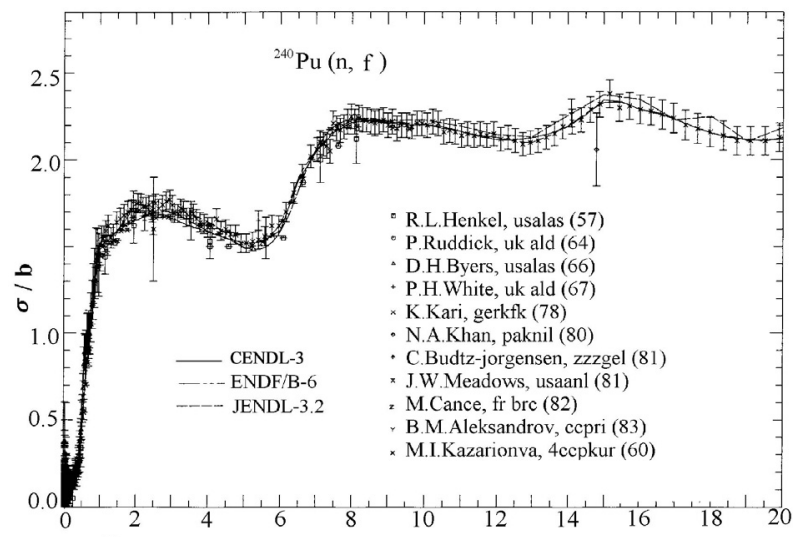

Fig. 9. ${ }^{240} \mathrm{Pu}(\mathrm{n}, \mathrm{f})$ evaluations compared with experimental data and other libraries.

JENDL and JEF-2.2 to validate CENDL-3.1 and to identify the source of the discrepancies with the experimental results.

\section{Light nuclides}

FNS experiments were used to validate $\mathrm{Be}, \mathrm{C}, \mathrm{N}, \mathrm{O}$ and $\mathrm{Li}$ evaluations. For $\mathrm{C}$, as shown in figure 11, good achievements were obtained in general, except discrepancy at 66.8 degree. For $\mathrm{LiO}_{2}$, good agreement with the benchmark values was achieved. Only a little under-prediction around $0.3 \mathrm{MeV}$ was observed in figure 12 , which was caused by the ${ }^{16} \mathrm{O}$ evaluation obviously.

\section{Medium-heavy nuclides}

Ti, Mn and Mo evaluations for CENDL-3.1 were tested with benchmark OKTAVIAN. From the comparison of neutron leakage current gives in figure 13, good agreement with the measurement above $0.8 \mathrm{MeV}$ was obtained for Ti. However, from the beginning to $0.8 \mathrm{MeV}$, the prediction is higher than the benchmark values. Since the evaluator of Ti confirmed $(\mathrm{n}, 2 \mathrm{n})$ cross section of ${ }^{48} \mathrm{Ti}$ is low enough, this discrepancy still can not be explained. For ${ }^{55} \mathrm{Mn}$ in figure 14 , an obvious under-prediction of neutron leakage around $10 \mathrm{MeV}$ occurs, 


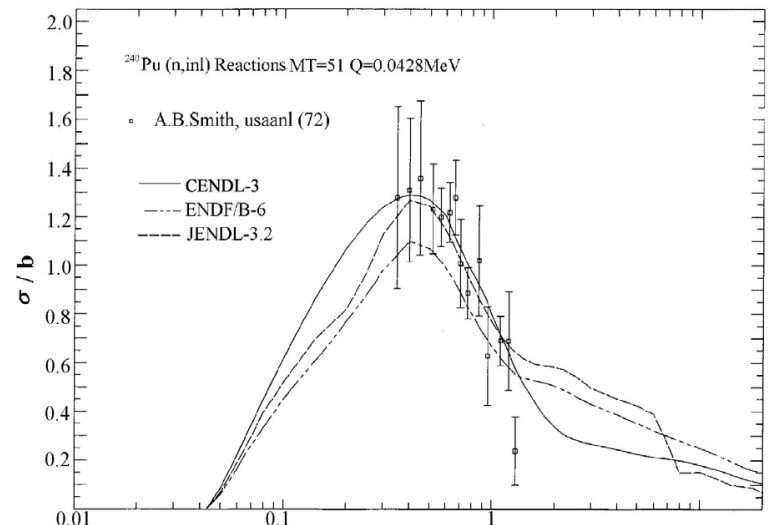

Fig. 10. ${ }^{240} \mathrm{Pu}(\mathrm{n}$,inel $)(\mathrm{MT}=51)$ evaluations compared with experimental data and other libraries.

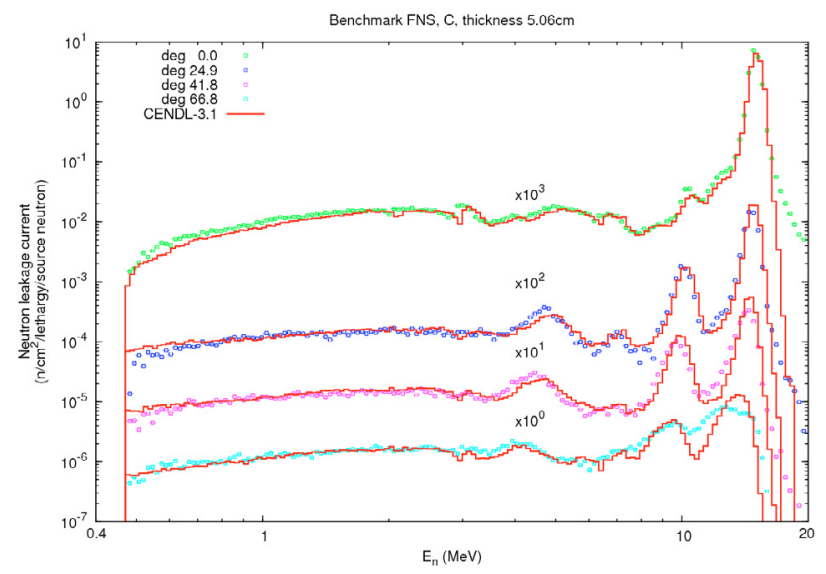

Fig. 11. Results for the neutron spectrum for the FNS C benchmark.

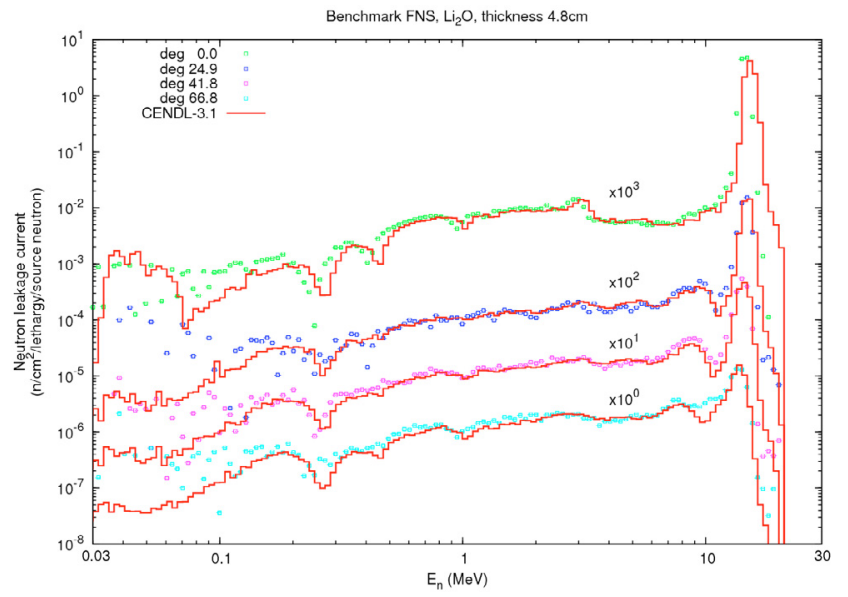

Fig. 12. Results for the neutron spectrum for the $\mathrm{FNS}_{\mathrm{LiO}}$ benchmark.

it is doubtable that direct reaction part of the inelastic cross section is missing. This problem should be corrected in future. For Mo, the calculated neutron leakage spectrum was lower than the measurement, especially from 6 to $13 \mathrm{MeV}$. Again, the contribution from inelastic scattering reaction is underestimated.

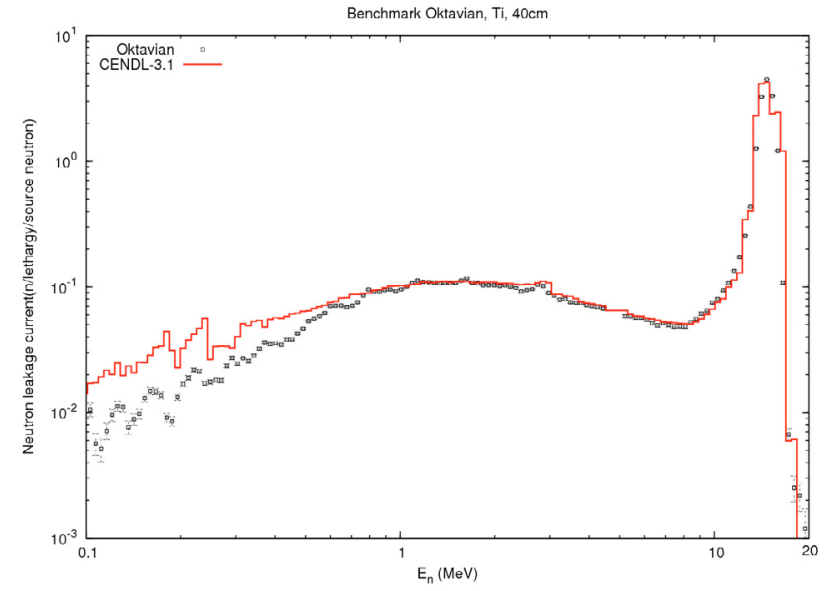

Fig. 13. Results for the neutron spectrum for the OKTAVIAN Ti benchmark.

\section{Actinides}

Tables 2 and 3 show the $K_{\text {eff }}$ results of calculation with different libraries of ${ }^{233} \mathrm{U}$ and ${ }^{238} \mathrm{U}$ as the examples.

\subsection{Conclusion}

1. CENDL-3.1 was finished in 2005, and it has been improved since then. CENDL-3.1 is being prepared for its release.

2. Nuclides and data files of CENDL-3.1 were increased and extended compared with CENDL-2.1.

3. All evaluations performed based on the new experimental data and new model calculations carefully. They were improved compared with CENDL-2.1.

4. The data of the most important nuclides are validated by the benchmark testing. Some of them are better than other evaluated libraries according to the results of the benchmark testing.

\section{Other progresses on nuclear data evaluations}

\subsection{Nuclear reaction model study}

The model was improved and completed for $1 \mathrm{p}$ shell light nuclides, which contains the dynamics and kinematics of nuclear reactions.

A method to set up file 6 of light nuclei for evaluated neutron data in ENDF/B-6 format below $20 \mathrm{MeV}$ has been established and the energy balance was strictly considered. This method has been used in the calculation of ${ }^{6,7} \mathrm{Li},{ }^{9} \mathrm{Be}$, ${ }^{10,11} \mathrm{~B},{ }^{12} \mathrm{C},{ }^{14} \mathrm{~N}$ and ${ }^{16} \mathrm{O}$.

The possibility of ${ }^{5} \mathrm{He}$ emission has been investigated in the light nuclear reactions, and the formulation for calculating of the ${ }^{5} \mathrm{He}$ emission was developed. Also the double differential cross sections of ${ }^{5} \mathrm{He}$ emission, as well as the spectra of neutron and alpha particle from the breakup of ${ }^{5} \mathrm{He}$, were set up and used in the series codes of LUNF. 


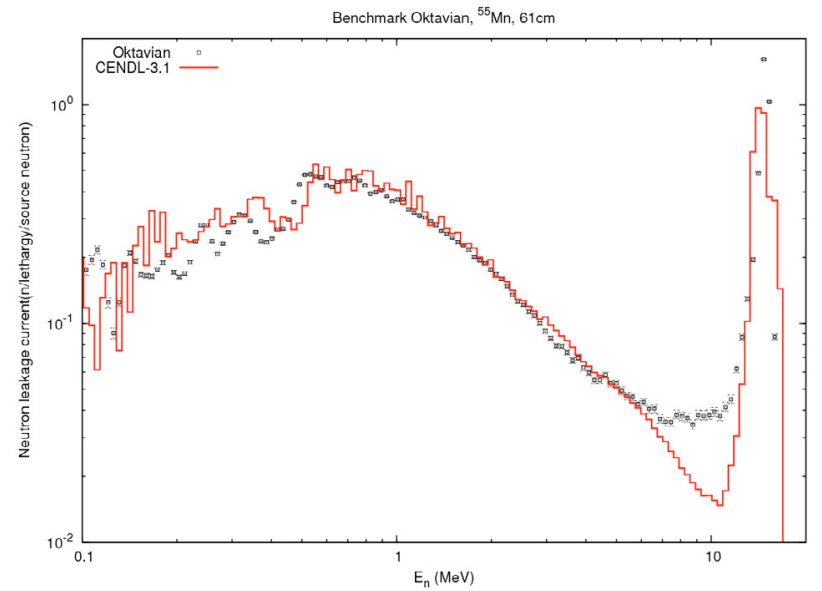

Fig. 14. Results for the neutron spectrum for the OKTAVIAN Mn benchmark.

Table 2. The calculations of $\mathrm{k}_{\mathrm{eff}}$ for ${ }^{233} \mathrm{U}$ benchmarks.

\begin{tabular}{lllll}
\hline Assembly & Experiment & CENDL-3.1 & ENDF/B-VI JENDL- \\
& & & & 3.2 \\
\hline${ }^{233}$ U Jezebel & $1.000(\sim 0.001)$ & 0.99822 & 0.99235 & 1.01460 \\
${ }^{233}$ U-F-002a & $1.000(\sim 0.001)$ & 0.99518 & 0.99544 & 1.00799 \\
${ }^{233}$ U-F-002b & $1.000(\sim 0.001)$ & 0.99583 & 0.99943 & 1.00959 \\
${ }^{233}$ U-F-003a & $1.000(\sim 0.001)$ & 0.99474 & 0.99755 & 1.01168 \\
${ }^{233}$ U-F-003b & $1.000(\sim 0.001)$ & 0.99523 & 0.99989 & 1.00929 \\
${ }^{233}$ U-F-004a & $1.000(\sim 0.001)$ & 1.00715 & 1.00494 & 1.01747 \\
${ }^{233}$ U-F-004b & $1.000(\sim 0.001)$ & 1.00808 & 1.00940 & 1.01721 \\
${ }^{233}$ U-F005a & $1.000(\sim 0.001)$ & 0.99951 & 0.99409 & 1.00852 \\
${ }^{233}$ U-F-005b & $1.000(\sim 0.001)$ & 1.00204 & 0.99803 & 1.00763 \\
Flattop-23 & $1.000(\sim 0.001)$ & 0.99218 & 1.00146 & 1.01140 \\
Averaging & $1.000(\sim 0.001)$ & 0.99882 & 0.99926 & 1.01154 \\
\hline
\end{tabular}

Table 3. The calculations of $\mathrm{k}_{\text {eff }}$ for ${ }^{238} \mathrm{U}$ benchmarks of thermal reactors.

\begin{tabular}{lllll}
\hline Assembly & CENDL-3.1 & JEF-2 & JENDL-3.2 & ENDF/B-6 \\
\hline TRX-1 & 0.9975 & 0.9952 & 0.9901 & 0.9908 \\
TRX-2 & 0.9998 & 0.9972 & 0.9920 & 0.9924 \\
BAPL-UO $_{2}-1$ & 1.0010 & 1.0020 & 0.9975 & 0.9952 \\
BAPL-UO $_{2}-2$ & 1.0003 & 1.0014 & 0.9972 & 0.9951 \\
BAPL-UO $_{2}-3$ & 1.0007 & 1.0014 & 0.9976 & 0.9960 \\
\hline
\end{tabular}

\subsection{Covariance study}

A code EXPOV for evaluating the covariance matrix of experimental data was developed. The covariance data are output in ENDF- 6 format. The code together with the spline fitting code SPC for multi-sets of correlative data was used to practically evaluate the covariance data for ${ }^{58,60,61,62,64, \text { nat }} \mathrm{Ni},{ }^{63,65, \text { nat }} \mathrm{Cu}$ and ${ }^{27} \mathrm{Al}$ and reasonable results have been obtained.

A program RAC based on the R matrix theory for calculating covariance data of light nuclide was developed. The program has been tentatively used to calculate the covariance data for ${ }^{6,7} \mathrm{Li},{ }^{10,11} \mathrm{~B}$, and ${ }^{16} \mathrm{O}$, reasonable results have been obtained for the cross sections up to $5 \mathrm{MeV}$. The calculated data and their covariance data for ${ }^{6} \mathrm{Li},{ }^{10} \mathrm{~B}$ have been accepted internationally as standard data of light nuclides.

Also a code has been developed for calculating the covariance data of structural material nuclides based on the statistical theory, including optical model, Hauser-Feshbach and preequilibrium emission model. The code has been used to calculate the covariance data of $\mathrm{Ni}, \mathrm{Cu}$ and their isotopes in ENDF-6 format.

\subsection{The systematic study of fission yield data}

Based on the mass distribution data up to $200 \mathrm{MeV}$ measured by Zoller, the systematic on dependence of chain yield on incident neutron energy for each mass number A was studied. And also the systematics of mass distribution on mass A and incident neutron energy was investigated by using 5 (or 3) Gaussian model. The calculated results could reproduce the experimental data used well. The investigation also shows that the correlation between the parameters of the systematic and the yields calculated with the systematics is quite complicated and, in general, is quite strong.

\subsection{The study on the dependence of yield on energy}

Taken some typical important fission products from ${ }^{235,238} \mathrm{U}$ fission, and the dependences of fission yield on incident neutron energy were studied. The covariance data were also evaluated based on each set of experimental data and the correlation among the data due to the systematical error, such as fission rate (or normalization), detector efficiency, decay data, etc., was taken into account in the fitting and the covariance matrix was obtained as a fit result. The results show that the data for most of product nuclides can be fitted with a linear function. But for some special product nuclides, the data have to be fitted with a spline function.

\subsection{Nuclear structure and decay data}

CNDC has taken permanent responsibility for evaluating and updating NSDD for $\mathrm{A}=51$, and 195-198 mass chain. The data have been revised using available experimental decay and reaction data for mass chain $\mathrm{A}=197$, and are being updated for mass chain $A=196$. Updated evaluation of $A=197$ has been sent to NNDC, USA and will be published in NDS in 2004. The evaluations of mass chain $\mathrm{A}=52-56$ were being updated at Jilin University. The decay data of ${ }^{233} \mathrm{U}$ were being evaluated on the basis of the new measured data.

\section{References}

1. Zhang Jingshang, Nucl. Sci. Eng. 142, 207 (2002).

2. Zhang Jingshan, Internal Report (2003).

3. RIPL http://www-nds.iaea.org/RIPL-2/.

4. Shen Qingbiao, CNDP (INDC(CRP)-053/L) 25, 19 (2001).

5. Shen Qingbiao, CNDP (INDC(CRP)-027/L) 7, 19 (1992).

6. J. Raynal, CEN-N2772 (1994).

7. Zhang Jingshan, Commun. Theor. Phys. (China) 39, 433 (2003).

8. Cai Conghai et al., Internal Report (1986).

9. Fusion Evaluated Nuclear Data Library CDROM, IAEA-NDSCD-6.

10. Y. Oyama et al., JAERI-M 94-014, 126 (1994).

11. C. Ichihara et al., JAERI-M 94-014, 125 (1994). 